\title{
Les cressonnières
}

\section{à l'origine de cas de distomatose humaine en Limousin}

\section{Etude des Mollusques vecteurs \\ et leur contrôle biologique}

\author{
par D. RONDELAUD \\ Laboratoire de Biologie animale $\left(P^{r} R\right.$. Sourie), Faculté des Sciences, F 87060 Limoges \\ et Laboratoire d'Histologie-Embryologie-Cytogénétique ( $P^{r}$ D. Barthe), Faculté de Médecine, \\ F 87032 Limoges.
}

\section{Résumé.}

Cette étude porte sur 16 cressonnières à l'origine de cas de distomatose humaine à Fasciola hepatica en Limousin. Deux espèces de Limnées: Lymnaea glabra et L. truncatula ont un rôle vicariant. La première espèce se rencontre isolée dans un tiers des cas.

L'introduction de Zonitoides nitidus, Mollusque prédateur, sans modification du milieu naturel permet l'élimination progressive des Limnées locales en 3 ans.

L'auteur insiste sur l'utilité de déterminer l'aptitude à la vicariance et ses limites chez L. glabra lorsque les 2 espèces: $L$. glabra et $L$. truncatula vivent dans les mêmes habitats.

\section{Summary.}

The water-cress pools in connection with cases of human fasciolasis in Limousin, France. Experimental studies of Trematode snails and their biological control.

Experimental studies on 16 water-cress pools with cases of human fasciolasis in Limousin were undertaken. Two species of snails, Lymnaea glabra and L. truncatula are here trematode hosts. L. glabra alone lives in 5 pools.

Accepté le 11 août 1978. 
The introduction of Zonitoides nitidus - predatory snail - without vegetation modification produces a progressive elimination of Lymnaea in 3 years.

The author insists on the use of determine the trematode host-aptitude of L. glabra and its limits when the two species - L. glabra and L. truncatula - live in the same habitats.

La plupart des cas de distomatose humaine à Fasciola hepatica L. sont dus principalement à l'ingestion de cresson (Nasturtium officinale). La contamination de cette plante est réalisée par l'intermédiaire des Limnées tronquées (Lymnaea truncatula Müller), hôtes intermédiaires habituels des formes larvaires de Fasciola. Aussi la prophylaxie de cette parasitose relève-t-elle de la protection sanitaire des cressonnières et de la destruction des Limnées (Ricou, 1966).

Nos investigations concernent des cressonnières naturelles non contrôlées et responsables de divers cas de distomatose humaine. Elles comportent les opérations suvantes :

1) Inventaire malacologique des' cressonnières.

2) Sensibilité à l'infestation expérimentale par les miracidiums de Fasciola des espèces de Limnées recensées.

3) Effets de la lutte biologique par l'introduction d'un Mollusque Pulmoné prédateur.

\section{Matériel et techniques}

\section{1. - Choix des cressonnières et inventaire de la faune malacologique.}

De 1971 à 1977 une enquête a été menée auprès des médecins généralistes ou des laboratoires d'analyses biologiques afin de rechercher des sujets ayant présenté dans les 15 années précédentes:

- soit les manifestations cliniques de la maladie (Symbole M dans le tableau I) ;

- soit quelques Fasciola adultes au niveau du foie ou de la vésicule biliaire lors d'interventions chirurgicales (Symbole C).

Les informations recueillies auprès de 41 personnes nous ont permis de retenir 16 cressonnières sauvages où la plante contaminée était réellement à l'origine d'infestations humaines.

L'inventaire malacologique porte sur les espèces et les nombres de Limnées présentes dans ou en bordure de la cressonnière. Nous avons également considéré les populations de Limnées vivant dans les prairies avoisinantes lorsque les cressonnières en reçoivent l'eau. S'il existe des Limnées infestées dans ces prairies, la contamination des cressonnières peut se faire par transport passif des cercaires. 
Tableau I. - Principales caractéristiques de 16 cressonnières à l'origine de cas de distomatose humaine étudiées entre 1972 et 1977

\begin{tabular}{|c|c|c|c|c|c|c|}
\hline $\begin{array}{c}\mathrm{s}^{0} \\
\mathrm{~d}^{\prime} \text { ordre }\end{array}$ & $\begin{array}{l}\text { Situation } \\
\text { géographique }\end{array}$ & $\begin{array}{c}\text { Type } \\
\text { (superficie } \\
\text { en } m 2 \text { ) }\end{array}$ & $\begin{array}{l}\text { Peuplement } \\
\text { malacologique } \\
\text { (Limées } \\
\text { seulement) }\end{array}$ & $\begin{array}{l}\text { Date de } \\
1 \text { 'infestation } \\
\text { bumaine } \\
\text { (Fasciota) }\end{array}$ & \multicolumn{2}{|c|}{$\overbrace{\text { Late de la târe année d'expérimentation }}^{\text {Etat de la cressonnière }}$} \\
\hline 1 & $\begin{array}{l}\text { Puy-de-Baneix } \\
\text { Jourgnac (87) }\end{array}$ & $\operatorname{II~}_{(4,8)}$ & $\begin{array}{l}\text { L. treroatuta } \\
\text { t. glabra }\end{array}$ & $\underset{M}{1967}$ & 1972 & Abandonnếe \\
\hline 2 & $\begin{array}{l}\text { Grumaud, } \\
\text { St-Symphorien-sur } \\
\text { Couze (87) }\end{array}$ & $\begin{array}{l}\text { II } \\
(3,7)\end{array}$ & L. glabru & $\underset{M}{1971}$ & 1972 & $\begin{array}{l}\text { Consomation } \\
\text { faniliale }\end{array}$ \\
\hline 3 & $\begin{array}{l}\text { Estivaux, } \\
\text { Veyrac (87) }\end{array}$ & $\stackrel{\text { I }}{{ }^{1}}$ & L. treneatula & ${ }_{M}^{1964}$ & 1973 & Abandonnée \\
\hline 4 & $\begin{array}{l}\text { St-victurnien } \\
(87)\end{array}$ & $\begin{array}{l}\text { II } \\
(7,9)\end{array}$ & L. glabra & $\underset{\mathrm{M}}{1959}$ & 1973 & $\begin{array}{l}\text { Consomation par } \\
\text { plusieurs familles }\end{array}$ \\
\hline 5 & $\begin{array}{l}\text { Rouffignac, } \\
\text { Meziêres/Issoire (87) }\end{array}$ & $\stackrel{1}{(7,2)}$ & L. glabma & ${ }_{c}^{1968}$ & 1973 & $\begin{array}{l}\text { Consommation } \\
\text { faniliale }\end{array}$ \\
\hline 6 & $\begin{array}{l}\text { ville jague } \\
\text { Marsac (23) }\end{array}$ & $\begin{array}{l}\text { II } \\
(3,8)\end{array}$ & L. trunaatula & $\begin{array}{c}1967 \\
c\end{array}$ & 1974 & Abandonnée \\
\hline 7 & $\begin{array}{l}\text { Le-Puy-du-Mont, } \\
\text { Aixe-sur-Vienne ( } 87 \text { ) }\end{array}$ & $\begin{array}{c}\text { III } \\
(19,3)\end{array}$ & L. tmonoatula & $\stackrel{1972}{M}$ & 1974 & Abandonnée \\
\hline 8 & Viam (19) & $\operatorname{II}_{(3,7)}$ & L. tmoneatula & ${ }_{c}^{1971}$ & 1974 & Abandonanée \\
\hline 9 & $\begin{array}{l}\text { Pierre-Buffière } \\
\text { (87) }\end{array}$ & $\mathrm{II}_{(8,2)}$ & L. gtabra & 1974 & 1974 & Abandonnée \\
\hline 10 & $\begin{array}{l}\text { Breuillaufa } \\
(87)\end{array}$ & $\stackrel{1}{1}$ & L. trencatula & $\begin{array}{c}1959 \\
\mathrm{H}\end{array}$ & 1974 & $\begin{array}{l}\text { Consomation } \\
\text { familiale }\end{array}$ \\
\hline 11 & $\begin{array}{l}\text { Marteneiche } \\
\text { Pontarion (23) }\end{array}$ & $\lim _{(7,8)}$ & $\begin{array}{l}\text { L. tmoreatula } \\
\text { i. glabra }\end{array}$ & $\begin{array}{c}1973 \\
\text { ou } 1974 \\
c\end{array}$ & 1975 & Abandonnĕe \\
\hline 12 & $\begin{array}{l}\text { La Graulière, } \\
\text { Saint-Yrieixe-sous } \\
\text { Aixe (87) }\end{array}$ & $\operatorname{IIII}_{(25,7)}^{\operatorname{III}}$ & $\begin{array}{l}\text { L. tmonoatula } \\
\text { L. glabra }\end{array}$ & (?) & 1975 & $\begin{array}{c}\text { Consonmation } \\
\text { familiale }\end{array}$ \\
\hline 13 & $\begin{array}{c}\text { La Croix d'Hervis } \\
\text { st-Priest-ligoure ( } 87)\end{array}$ & $\stackrel{\text { I }}{(12,3)}$ & - & $\begin{array}{c}1967 \\
M\end{array}$ & 1976 & Vente locale \\
\hline 14 & $\begin{array}{l}\text { La Mazière, } \\
\text { Royères (87) }\end{array}$ & $\mathrm{II}_{(11,3)}$ & $\begin{array}{l}\text { L. tmoroatula } \\
\text { L. palustrvie }\end{array}$ & $\underset{c}{1971}$ & 1976 & Abandonnée \\
\hline 15 & $\begin{array}{l}\text { Les Champs, } \\
\text { Champnétery (87) }\end{array}$ & $\operatorname{III}_{(80,7)}$ & L. tmonoatula & $\underset{\mathrm{M}}{1975}$ & 1976 & Abandonnée \\
\hline 16 & $\begin{array}{l}\text { Fregeaigue, } \\
\text { Nantiat (87) }\end{array}$ & $\operatorname{III}_{(3,4)}$ & L. glabra & $\underset{c}{1975}$ & 1977 & $\begin{array}{l}\text { Consomation } \\
\text { familiale (?) }\end{array}$ \\
\hline
\end{tabular}

\section{2. - Technique parasitologique.}

La méthode utilisée dérive des données fournies par les expériences de Boray $(1966,1967)$ : la plupart des espèces de Limnées voisines de Lymnaea truncatula ne sont infestables que très jeunes, nouvellement écloses.

Pour chaque espèce de Limnées identifiée par cressonnière, plusieurs individus adultes sont récoltés lors de la première année d'observation et placés dans des bacs d'élevage soumis aux conditions standard du laboratoire (Rondelaud, 1974). Les jeunes issus des pontes sont soumis dans les 24 heures à une masse de miracidiums juste éclos, puis ils sont gardés en élevage pendant 6 semaines à $20^{\circ} \mathrm{C}$, par groupe de 500 pour chaque espèce de Limnées.

A la $6^{\circ}$ semaine, les nombres d'individus morts et de Limnées présentant une infestation mature (cercaires) par rapport à l'effectif initial caractérisent la sensibilité de chaque population de Limnées à une infestation miracidienne.

\section{3. - Technique de lutte biologique.}

Le Pulmoné prédateur terrestre : Zonitoides nitidus (Rondelaud, 1978) a été introduit, à raison de 10 individus au mètre carré, sur les berges des cressonnières lors du . retrait partiel de l'eau durant les mois d'été. 
Les effets des prédateurs ont été suivis pendant 4 ans par comptage des effectifs des diverses populations de Limnées et de prédateurs juste avant l'exondation estivale du cresson.

\section{Les caractéristiques de quelques cressonnières en Limousin}

Nous avons indiqué, sur le tableau I, les principales caractéristiques des 16 cressonnières prospectées. Ces dernières sont situées :

- soit en dérivation $\left(\mathrm{n}^{\mathrm{os}} 3,5\right.$ et 10$)$, soit sur le cours même d'un ruisseau à courant rapide $\left(\mathrm{n}^{\circ} 13\right)$ (type $\left.\mathrm{I}\right)$;

- soit à l'émergence de sources situées à flanc de côteau (type II) dans des emplacements divers (pêcheries, fossés, rigoles...). La superficie est toujours réduite (moins de $20 \mathrm{~m}^{2}$ );

- soit dans des prairies marécageuses dans le fond des vallées (type III). La superficie est alors parfois vaste $\left(n^{\circ} 15\right)$.

Aucune cressonnière n'est entretenue.

Il faut remarquer que malgré l'existence de cas de distomatose humaine, le cresson faisait encore l'objet de consommation familiale dans 6 cas et était vendu sur le marché local dans 1 cas, tout au moins lors de la $1^{\text {re }}$ année d'expérimentation.

\section{Résultats et interprétation}

\section{1. - Inventaire des Limnées dans les cressonnières explorées.}

Trois espèces ont été reconnues. L'une d'elles, Lymnaea palustris, n'a été vue qu'une fois et en faible effectif sur une cressonnière de type II. Etant donné de plus que cette espèce résiste aux essais d'infestations, il n'en sera pas autrement question.

Les 2 autres espèces, L. truncatula et $L$. glabra ont été trouvées en peuplements monospécifiques ou associées (fig. 1).

L. glabra domine en nombre dans les stations du type II, $L$. truncatula sur celles du type III.

Il n'a pas été trouvé de Limnées dans la localité 13. La constatation d'un cas de distomatose humaine s'y rapportant est peut-être à imputer à la présence de ces Mollusques à $120 \mathrm{~m}$ en amont, sur le ruisseau.

2. - Infestations expérimentales des Mollusques nouveau-nés par les miracidiums.

Sur la figure 2 sont indiqués les pourcentages de Limnées présentant une infestation mature (avec cercaires) au bout de 6 semaines d'élevage. Les lots de jeunes individus proviennent de souches recueillies dans les 16 localités explorées.

Le taux moyen d'infestation de Lymnaea truncatula est plus de 3 fois celui de L. glabra. Cependant, dans le cadre de nos investigations en Limousin, des cas de 


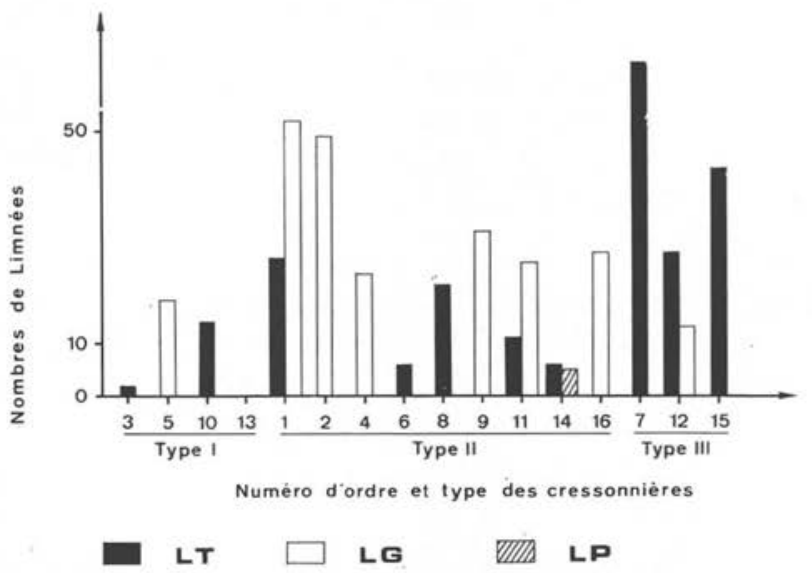

Fig. 1. Inventaire malacologique des 16 cressonnières prospectées lors de la première année d'étude.

L.T. : Lymnaea truncatula; L.G.: L. glabra ; L.P.: L. palustris.

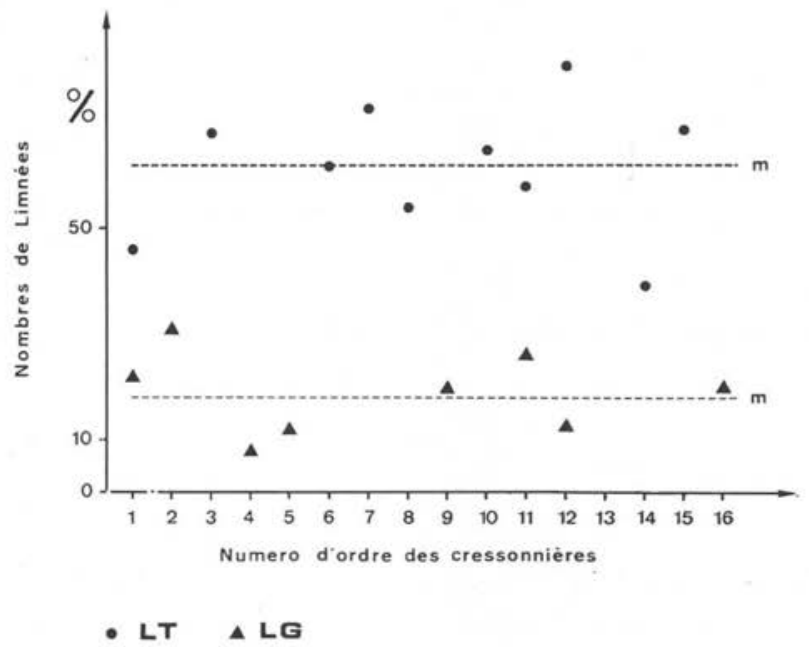

Fig. 2. Les effectifs de Limnées (en $\%$ ) présentant des cercaires indépendantes au bout de 6 semaines d'infestation.

L.T. : Lymnaea truncatula ; L.G.: L. glabra ; $m$ : taux moyen d'infestation.

distomatose humaine ont été relevés qui peuvent être mis en rapport avec la présence de cette seule espèce (tableau $I$, localités $2,4,5,9,16$ ).

Aussi, contrairement à l'opinion courante, L. glabra pourrait être vecteur accessoire de la fasciolose. 
3. - Lutte biologique dans les cressonnières.

Nos résultats (tableau II) ne portent que sur 7 cressonnières pendant 4 ans avec introduction de Zonitoides nitidus lors de l'assèchement estival du cresson sans fauche. Nous remarquons l'existence d'une diminution progressive des effectifs de Limnées au fur et à mesure des années avec disparition complète en 3 ans.

Tableau II. - Les résultats fournis par l'introduction de Zonitoides à raison de 10 par $\mathrm{m}^{2}$ d'habitat sur 7 cressonnières pendant 4 années.

\begin{tabular}{|c|c|c|c|c|c|c|c|c|c|}
\hline \multirow[b]{2}{*}{$\mathrm{N}^{*} \mathrm{~d}^{\prime}$ ordre } & \multicolumn{9}{|c|}{$\begin{array}{l}\text { Nombres de Limnées } \\
\text { (Nombres de prédateurs) }\end{array}$} \\
\hline & $\begin{array}{l}\text { Espèces } \\
\text { de Limnées }\end{array}$ & & & & née & & & & anée \\
\hline 6 & L. trosoatula & 7 & (38) & 4 & (17) & 1 & (13) & - & $(-)$ \\
\hline 7 & L. tronoatula & 63 & (193) & 32 & (83) & 7 & (47) & -2 & (13) \\
\hline 8 & L. trioroatula & 21 & (37) & 11 & (73) & - & (56) & - & (3) \\
\hline 9 & L. glabra & 31 & (82) & - & (32) & - & (9) & - & $(-)$ \\
\hline 10 & L. truncatula & 14 & (37) & 2 & (119) & - & (36) & - & (11) \\
\hline 11 & $\begin{array}{l}\text { 2. trinoatula } \\
\text { L. glabra }\end{array}$ & $\begin{array}{l}11 \\
25\end{array}$ & (78) & $\begin{array}{r}3 \\
17\end{array}$ & (56) & $\overline{2}$ & (23) & $\begin{array}{l}- \\
-\end{array}$ & (17) \\
\hline 12 & $\begin{array}{l}\text { L. truncatula } \\
\text { L. glabra }\end{array}$ & $\begin{array}{l}27 \\
13\end{array}$ & (257) & $\begin{array}{r}2 \\
25\end{array}$ & (43) & - & (7) & - & (9) \\
\hline
\end{tabular}

La reproduction des prédateurs introduits est possible mais on assiste à une décroissance régulière des nombres de descendants, plus lente cependant que celles des Limnées.

\section{Discussion}

Le problème des Mollusques vecteurs ou non des formes larvaires de Fasciola hepatica constitue une voie de recherche encore relativement vierge, malgré l'existence de nombreuses références bibliographiques. L'examen de ces travaux (Euzeby, 1971) montre :

- que dans la plus grande partie de l'Europe, la Limnée tronquée est à peu près la seule espèce apte à permettre dans les conditions naturelles l'évolution larvaire du parasite ;

- que d'autres espèces: L. palustris, L. glabra, L. stagnalis - lorsqu'elles sont infestées jeunes dans les conditions du laboratoire - jouent le même rôle de vecteur intermédiaire ; 
- que l'absence de la Limnée tronquée peut être palliée dans les conditions naturelles par l'intervention d'espèces voisines (L. peregra, L. ovata et $L$. occulta).

Il existe donc une discordance entre les résultats de l'expérimentation et les observations réalisées dans les conditions naturelles.

Le travail présenté ci-dessus ne porte que sur 16 cressonnières reconnues à l'origine de cas de distomatose humaine en Limousin. Les résultats montrent la fréquence des populations de L. glabra dans ces biotopes et la possibilité pour cette espèce d'être un vecteur potentiel de Fasciola hepatica.

Nous formulons cependant quelques réserves vis-à-vis de ces résultats :

- L'information venant des personnes ayant eu la distomatose peut se révéler entachée d'une certaine imprécision concernant la nature de la plante consommée et son origine, compte tenu de la réputation locale qu'a la distomatose d'être une «maladie gênante ». Cette éventualité est très faible, en raison de notre sévérité dans la sélection des 16 cressonnières d'expérimentation.

- Il existe toujours un intervalle de temps entre la consommation du cresson contaminé et le début de notre prospection dans la cressonnière en cause. Pour les localités à $L$. glabra seules, cet intervalle varie de 4 mois $\left(n^{\circ} 9\right)$ à 14 ans $\left(n^{\circ} 4\right)$. Il est logique d'évoquer la possibilité d'une disparition de Limnées tronquées dans ces localités au cours de cet intervalle de temps. Mais cette possibilité est assez peu probable, surtout dans les cressonnières à intervalle de temps court $\left(\mathrm{n}^{\circ \mathrm{s}} 2,9\right.$ et 16).

Lymnaea glabra est considérée par nombre d'auteurs comme une espèce pouvant jouer un rôle de vecteur en l'absence de la Limnée tronquée. Il conviendra d'établir expérimentalement les taux d'infestation de peuplements mixtes à $L$. glabra et $L$. truncatula pour déterminer avec précision les aptitudes à la vicariance de la première espèce.

\section{REMERCIEMENTS}

L'auteur exprime sa gratitude aux médecins et aux directeurs de laboratoires d'analyses pour leur aide dans cette enquête, notamment $\mathbf{M}$. le $\mathrm{D}^{r}$ Hervy de Limoges, M. Leymarie de Brive.

\section{Bibliographie}

Boray J.C. (1966): Studies on the relative susceptibility of some Lymnaeids to infection with Fasciola hepatica and F. gigantica and on the adaptation of Fasciola spp. Ann. Trop. Med. Parasit., 60, 114-124.

Boray J.C. (1967) : Host-parasite relationships between Lymnaeid snail and Fasciola hepatica. In: "The reaction of the host to parasitism ", N.G. Elwert Universitats- und Verlagsbuchhandlung, édit., Marburg-Lahn, 132-140.

Euzéby J. (1971): Les maladies vermineuses des animaux domestiques et leurs incidences sur la pathologie humaine. Tome II : Maladies dues aux Plathelminthes. $2^{\circ}$ fasc.: Trématodes. Livre 1. Vigot frères, édit., Paris, 799 p. 
Ricou G. (1966) : Les mesures prises en France. Colloque d'information scientifique sur les animaux nuisibles dans les cressonnières. C.R.A. Gembloux, édit., Gembloux, 68-74.

Rondelaud D. (1974): Recherches sur l'influence de quelques facteurs physiques sur les migrations quotidiennes de Galba truncatula Müller. Ann. Parasitol. Hum. Comp., 49, 417-425.

Rondelaud D. (1978) : Contribution à l'étude écologique et éthologique de Lymnaea (Galba) truncatula Müller, vecteur de Fasciola hepatica L. Recherche de moyens de lutte biologique en Limousin. Thèse Doct. ès Sci. Nat., n ${ }^{\circ} 78-4$, Limoges, 302 p. 